\title{
TERAPEUTAS OCUPACIONALES: INFLUENCIA DEL GÉNERO EN SU DESEMPEÑN LABORAL
}

\author{
OCCUPATIONAL THERAPIES: GENDER INFLUENCE IN THEIR WORK PERFORMANCE
}

\author{
María Isabel Mansilla Rivera ${ }^{1}$, Natalia Cristina Astete Valdebenito² ${ }^{2}$, Leticia Catherine \\ Garrido Salazar ${ }^{3}$ y Felipe Cristóbal Maldonado Molina ${ }^{4}$
}

\section{Resumen}

Esta investigación se fundamenta desde una perspectiva y enfoque de género. Su objetivo fue analizar la influencia del género en el desempeño laboral de los Terapeutas Ocupacionales en la ciudad de Temuco, basándose en las percepciones y vivencias de dichos profesionales.

La metodología utilizada corresponde al enfoque cualitativo, utilizando como sustento teórico la fenomenología. La muestra del estudio fue intencional y estuvo constituida por 10 profesionales, siendo el número de hombres y mujeres iguales en cantidad. Se utilizó la entrevista semiestructurada como instrumento de recolección de datos analizando la información obtenida a través del análisis de contenido y análisis de discurso. Los tópicos abordados consideran la relación entre trabajo y género y hacen referencia a: diferencias salariales, costos laborales, manejo de poder y estatus de minoría.

Los resultados obtenidos señalan que existe influencia del género en el desempeño laboral de los Terapeutas Ocupacionales en todas las determinantes analizadas, principalmente en costos laborales y estatus de minoría. Con respecto al impacto en el desempeño existen opiniones divididas, ya que el $80 \%$ de la muestra femenina considera que existe influencia de género en el desempeño laboral, frente al $60 \%$ masculino que no visualiza inluencias en su quehacer de terminados por la variable de genero. Sin embargo, se hace necesario destacar que lo anterior no está determinado por la disciplina, sino que por las construcciones culturales de la sociedad, por lo que debemos ser más críticos con los fenómenos derivados de género, considerando que es una categoría de análisis inherente al ser humano.

\section{Palabras claves}

Género, Desempeño laboral, Terapeutas Ocupacionales

\footnotetext{
Licenciada en Terapia Ocupacional otorgado por la Universidad de La Frontera, Terapeuta Ocupacional, E-mail: Isabel.mansillarivera@gmail. com, +56968133723, Researched ID: E-7803-2017, ORCID iD: 0000-0001-6293-6312, Código postal: 5700647

Licenciada en Terapia Ocupacional otorgado por la Universidad de La Frontera, Terapeuta Ocupacional, E-mail: N.astetevaldebenito@gmail. com, +56992632693, Researched ID: E-7815-2017, ORCID iD: 0000-0002-4119-4345, Código postal: 4803225.

Licenciada en Terapia Ocupacional otorgado por la Universidad de La Frontera, Terapeuta Ocupacional, E-mail: Leticia.garridosalazar@ gmail.com, +56992530482, Researched ID: E-8487-2017, ORCID iD: 0000-0002-3079-2083, Código postal: 4781056.

Estudiante de Terapia Ocupacional de la Universidad de La Frontera, E-mail: Fcristobalmm@gmail.com, +56971882845, Researched ID: E-8394-2017, ORCID iD: 0000-0001-6030-0273, Código postal: 4850722.
} 


\section{Abstract}

This research is based on a gender perspective and approach. Its objective was to analyze the gender's influence on the performance of Occupational Therapists's work in the city of Temuco, based on the perceptions and experiences of these professionals.

The methodology used corresponds to the qualitative approach, using the phenomenology as theoretical support. The sample of the study was intentional and was constituted by 10 professionals, being the number of men and women equal in quantity. The semistructured interview was used as an instrument of data collection by analyzing the information obtained through content analysis and discourse analysis. The topics covered consider the relationship between work and gender and refer to: wage differences, labor costs, power management and minority status.

The results obtained indicate that there is gender influence in the occupational therapists' work performance in all the determinants analyzed mainly in labor costs and minority status. With regard to the impact on performance, there are divided opinions, since $80 \%$ of influ he discipline, but by the cultual consuctions of he socion gender, considering that it is a category of analysis inherent to the human being.
\end{abstract}

\title{
Key words
}

Gender, work performance, Occupational Therapists

Recepcionado: 30/3/2017

Aceptado: 19/06/2017

\section{INTRODUCCIÓN}

A lo largo de la historia se han suscitado una serie de problemáticas asociadas al género, confluyendo elementos socio-políticos de diversa índole: sexualidad, políticas públicas, trabajo remunerado, trabajo doméstico, participación ciudadana y organización social (Chávez, 2004).

Considerando que estas problemáticas están presentes en la sociedad, surge la siguiente interrogante: ¿Por qué existen estas problemáticas derivadas de género?

En un estudio realizado por la Asociación de Hombres por la Igualdad de Género (AHIGE, 2011), se afirma que:

Históricamente, la sociedad ha dividido a las personas según el sexo con que nacían, asignándoles roles e identidades culturales (de género) distintas y no equiparables en derechos y oportunidades. En esta sociedad patriarcal, las mujeres han sufrido una grave discriminación estructural que aún perdura, utilizando en muchos casos nuevas formas, hasta nuestros días.

El estudio de Intervención Social desde el enfoque de género (2010), apoya la idea anterior, afirmando que el modelo patriarcal predominante en la sociedad, realiza una distinción entre las funciones que ejercen ambo géneros, sin tener en consideración sus capacidades ni conocimientos, sino solo la condición de ser hombres o mujeres.

Sobre la base de lo anterior, según lo referido por Facio y Fries (2005), el sistema patriarcal no sólo explica y construye las desigualdades entre mujeres y hombres, sino que conserva y agudiza otras formas de dominación.
Por otra parte, se evidencia la existencia de profesiones en las que predomina un género por sobre otro. Según se menciona en el estudio Masculinity at Work: The Experiences of Men in Female Dominated Occupations de Simpson (2004), los hombres y mujeres se mueven cada vez más en áreas atípicas de género. Estas áreas hacen alusión a espacios donde se desenvuelve una cantidad mayoritaria de un género por sobre otro (Hakim, 2000, citado de Simpson, 2004). Es así como este fenómeno se puede observar en el área de la salud, en donde han existido carreras en que el número de mujeres es ampliamente mayor al de varones (Departamento de Estadísticas e Información de Salud, 2012).

De esta forma, en el contexto de la carrera de Terapia Ocupacional, según el estudio realizado por el Colegio de Terapeutas Ocupacionales de Chile (COLTO, 2014), acerca de la cantidad de Terapeutas Ocupacionales registrados en la Superintendencia de Salud en el año 2012, se observa una clara predominancia del género femenino $(80 \%)$ por sobre el masculino $(20 \%)$ en el ejercicio de la profesión.

Al existir estas diferencias entre los Terapeutas Ocupacionales, surgen las inquietudes de conocer ¿qué asimetrías relacionadas al género se producen en el área laboral de los Terapeutas Ocupacionales? En el caso de existir estas determinantes, ¿afectarán el desempeño laboral de dichos profesionales? 
Para responder estas interrogantes, se llevará a cabo un estudio cualitativo, donde se recogerán las percepciones y vivencias basadas en las experiencias de los participantes, Terapeutas Ocupacionales que se desempeñen en la ciudad de Temuco, analizando cuatro determinantes que se desprenden de género y área laboral, las cuales consisten en: diferencias salariales, costos laborales, manejo de poder y estatus de minoría.

\section{MéTOdo}

\section{A) Diseño}

El enfoque de esta investigación es de carácter cualitativo, de tipo fenomenológico. Este tipo de estudio utiliza técnicas de recolección de datos que indagan en situaciones naturales intentando dar sentido o interpretar los fenómenos en los términos del significado que las personas les otorgan \{(Denzin y Lincoln, 1994) citado por Vasilachis 2009)\}.

El método de recolección de información fue a través de entrevistas semiestructuradas.

Para la producción de resultados se utilizó la técnica análisis de contenido, así como también la técnica de análisis del discurso.

\section{B) Población y muestra}

Para la presente investigación, los autores utilizaron el muestreo intencionado, con un total de 10 Terapeutas Ocupacionales, 5 hombres y 5 mujeres que ejercen en la ciudad de Temuco, y que cumplían los siguientes criterios:

- Profesionales Terapeutas Ocupacionales. (Que se desempeñen como Terapeuta Ocupacional).

- Haber egresado de la carrera de Terapia Ocupacional en un tiempo igual o mayor a un año.

- Tener por lo menos un año de experiencia en el ejercicio de la profesión.

- Que ejerzan la profesión en la ciudad Temuco con un mínimo de 6 meses.

Tabla 1: Caracterización de la muestra

\begin{tabular}{|l|l|l|l|l|}
\hline Participante & Edad & Institución & Año de egreso & $\begin{array}{l}\text { Comuna de } \\
\text { residencia }\end{array}$ \\
\hline Entrevistado $n^{\circ} 1$ & 37 & Cecosam Amanecer - UCT & 2004 & Temuco \\
\hline Entrevistado $n^{\circ} 2$ & 33 & UCT - UCI HHHA & 2005 & Temuco \\
\hline Entrevistado $n^{\circ} 3$ & 63 & U Autónoma & 1966 & Temuco \\
\hline Entrevistado $n^{\circ} 4$ & 33 & U Autónoma & 2006 & Temuco \\
\hline Entrevistado $n^{\circ} 5$ & 61 & IP Chile & 1976 & Temuco \\
\hline Entrevistado $n^{\circ} 6$ & 28 & Teletón Temuco & 2011 & Temuco \\
\hline Entrevistado $n^{\circ} 7$ & 35 & U Mayor & 2008 & Temuco \\
\hline Entrevistado $n^{\circ} 8$ & 28 & U Autónoma & 2012 & Temuco \\
\hline Entrevistado $n^{\circ} 9$ & 37 & U Autónoma & 2004 & Temuco \\
\hline Entrevistado $n^{\circ} 10$ & 29 & U Mayor - Hospital de Pitrufquén & 2009 & Temuco \\
\hline
\end{tabular}




\section{C) Consideraciones éticas}

Los participantes no se vieron afectados de manera negativa en el ámbito psicológico, físico ni moral, debido a que se resguardo su identidad y el proceso investigativo se llevó a cabo con total confidencialidad, respetando así, el principio ético de no maleficencia, beneficencia justicia y autonomía. Así mismo cada participante, través de un consentimiento informado fue notificado de manera oportuna sobre las implicancias y objetivos de la investigación, además se les dio la posibilidad de participar o no de la investigación y el derecho de poder retirarse en cualquier momento del estudio, si así lo deseaba.

\section{RESULTADOS}

\section{Diferencias salariales}

En cuanto a esta categoría de análisis, la que hace referencia a la diferencia existente entre los salarios percibidos por los trabajadores de ambos sexos (Unión Europea, 2014), el 90\% de los Terapeutas Ocupacionales entrevistados menciona que no ha experimentado en su quehacer profesional diferencias en su remuneración dadas por el género, indicando que tales diferencias responden a factores relacionados a la capacidad de negociación la experiencia laboral y el grado al que pertenecen en el servicio público.

Con respecto a la capacidad de negociación, la Profesora Rosario Macera de la Universidad Católica de Chile (2015) señala que las mujeres raramente negocian, al contrario de lo que sucede con los hombres, quienes $\mathrm{s}$ negocian su sueldo, siendo percibidos como ambiciosos, exitosos y por consiguiente, valiosos para la organización.

Lo anterior concuerda con lo expresado por el $20 \%$ de los entrevistados, quienes mencionan que según su percepción, las mujeres negocian menos su sueldo a diferencia de lo que ocurre con sus colegas varones.

"No, no me he encontrado casos así como diferencias salariales. Lo que sí he encontrado, de repente es, como el tema de la negociación (...) Algunas colegas mujeres como que les cuesta el tema de negociar decir pucha, yo quiero un poquito más" (Ent: 7)
Por otro lado, el 30\% de la muestra refiere que las diferencias salariales están determinadas por la experiencia laboral que se posea más que por un tema de género o que se asocia a especializaciones de postgrados y/o magister.

"Yo esperaría que alguien que tiene más formación, más experiencia, sin desmerecer a los recién egresados, eso yo creo que debiera estar reflejado en la remuneración y la responsabilidad del cargo, más allá de si eres hombre o mujer" (Ent: 4)

"Lo que sí puede influenciar son los magister que hay de por medio, eso te paga un poco más, pero eso en relación al grado académico que uno pueda tener, pero en relación al género, no he visto mayores diferencias (Ent: 8)

No obstante, según lo relatado por el $10 \%$ de la muestra entrevistada, existen diferencias salariales según el área en que se desempeñen los Terapeutas Ocupacionales en su trabajo.

"Sé que en algunas áreas uno gana mejores sueldos, pero tiene que ver con las áreas más que por género" (Ent: 1)

Sin embargo y en contraste con los relatos anteriores, $10 \%$ de los participantes señala haber vivenciado brechas en los ingresos por un tema de género.

"Si, si en casi todos los trabajos en los que he estado se ha percibido esa diferencia de que el hombre gana más plata que la mujer, haciendo el mismo cargo (...) En alguno trabajos que he tenido ha sido muy notorio, ha sido el $50 \%$ más por ejemplo, el $30 \%$ más y en otros ha sido menos" (Ent: 2 )

Con respecto al impacto en el desempeño laboral, los entrevistados manifiestan que para quienes experimentan estas diferencias, influyen principalmente a nivel de disposición, compromiso y aspecto anímico en el trabajo 


\section{Costos laborales}

Este apartado hace alusión a la suma de costos monetarios y no monetarios por parte del empleador para contratar y mantener el empleo de un trabajador (Todaro, Godoy y Abramo, 2001). En general, la idea de que los costos laborales de las mujeres son más altos que los de los hombres incide significativamente en sus procesos de toma de decisión. Estos supuestos costos más elevados estarían relacionados básicamente con los mecanismos legales de protección a la maternidad, tales como los permisos pre y postnatal, el horario especial para la lactancia, los permisos para el cuidado de hijos enfermos, la estabilidad para la mujer embarazada o en el período post parto y los servicios de cuidado infantil (Todaro, Godoy y Abramo, 2001).

En el presente estudio se consideraron los costos laborales que incluyen los permisos de pre y post natal, capacitaciones, accidentes laborales y enfermedades profesionales. En base a lo anterior, el $80 \%$ de la muestra afirma que el permiso de pre y post natal en el caso de la mujer, es el que más influye en su desempeño laboral, señalando también que se produce una incompatibilidad entre el rol de madre y trabajadora, destacando la idea de que la mujer es más cara que el hombre en términos de costos.

"Yo pienso que sí, fundamentalmente en el pre y post natal yo creo que sí, eso me parece que tiene una influencia fuerte, lo he oído comentar a gente que no va a contratar a una mujer porque se casó hace poco por ejemplo y lo más probable es que tenga un hijo luego" (Ent: 2)

"Ahora en la realidad actual pareciera ser que la mujer tiene más costos, por lo tanto estadísticos de incompatibilidad de rol de madre y trabajadora (...) ciertos costos asociados que quizás la mujer es más cara que un hombre, así súper concreto es más cara para un empleador que un hombre" (Ent: 4)

En el caso del pre y post natal la influencia en el quehacer profesional, se verá reflejado en las proyecciones laborales en el caso de las mujeres y en el equipo de trabajo por la reorganización de roles y horarios de trabajo desde el punto de vista de hombres, viendo este tema asociado a la maternidad como un proceso negativo más que un componente natural.
Por otra parte, en el caso de las capacitaciones, accidentes laborales y enfermedades profesionales, los entrevistados plantean que es transversal tanto para hombres como para mujeres y que afectan de igual manera independientemente del género.

\section{"No, es parejo, no me parece que responde al género (...) es transversal (...) y común- mente son por acciones que no se hacen bien" (Ent: 6)}

Sin embargo los participantes de la muestra de estudio también mencionaron otros factores que consideran influyentes en el desempeño de los Terapeutas Ocupacionales en el área laboral. Estos factores corresponden a hostigamiento laboral, tipos de contrato, el contexto y el riesgo del puesto de trabajo. En este sentido el $10 \%$ de la muestra refiere haber presenciado actos de este tipo.

"Lo que he percibido es acoso laboral, por parte de algunas compañeras de trabajo... que han tenido hace poquito, hijos, y están con el fuero maternal" (Ent. 8)

En relación a los tipos de contrato, según el Estatuto Administrativo existen funcionarios de planta, funcionarios a contrata y funcionarios a honorarios. De acuerdo a lo anterior, el $10 \%$ de los entrevistados afirma que los costos laborales van a depender del tipo de contrato que se posea.

"Tu obtienes más beneficios dependiendo del tipo de contrato que tienes (...) la diferencia estaba más que nada en la calidad contractual" (Ent: 1)

En cuanto al contexto, según la AOTA (2010), el término hace referencia a "la variedad de condiciones interrelacionadas que están dentro y rodeando al cliente" (p. 28 - 29).

Según lo antes expuesto, el 10\% de los profesionales entrevistados expresa que el contexto de trabajo está directamente relacionado con los costos y con los riesgos laborales que cada puesto de trabajo pueda tener asociado.

"Es distinto trabajar en cárcel a trabajar con los jóvenes en la esquina, o trabajar en Teletón o trabajar haciendo docencia, 
el contexto, las situaciones, los riesgos a los que estás expuesto, (...) son muy distintas en uno u otro" (Ent: 4)

\section{Manejo de poder}

Este punto guarda relación con los cargos directivos y acceso a los puestos de trabajo en Terapia Ocupacional. Varios estudios señalan la presencia de significativas barreras para el acceso de mujeres a los puestos gerenciales, es decir, "obstáculos artificiales e invisibles basados en prejuicios psicológicos y estructurales" (OIT 1997, citado por Todaro, Godoy y Abramo 2001), que limitan el ingreso a cargos de alto nivel y definen ciertos patrones de inserción.

En relación al primer término el 70\% de los entrevistados manifestó que no existe influencia del género para asumir cargos de poder, ya que se le da más importancia a las experiencias y competencias profesionales que posean.

"Quienes acceden finalmente a puestos más de poder son también las que tienen más experiencias y la experiencia también está dada por los años" (Ent: 9)

"Creo que lo más fundamental o lo que se logra pedir son las habilidades, las competencias profesionales y las competencias éticas que pueda tener la persona" (Ent: 8)

Sin embargo y en contraste con lo anterior, el 30\% de la muestra percibe que si existe una influencia debido al género para asumir cargos directivos.

"Yo creo que si en general se tiende a poner en cargos directivos a los hombres, porque se piensa que son menos emocionales, que tiene más posibilidad de dirigir gente con más seriedad, esos son como estereotipos que se adjudican más como al hombre y no a la mujer" (Ent: 2)

"Lo que yo logro visualizar es que pareciera ser que predomina el género masculino en cargos de poder administrativo, financiero toma de decisiones me parece que hay mayor cantidad de hombres aun cuando son pocos en la carrera ellos toman cargos que son más, o no sé ¿Quiénes publican más?" (Ent: 4
En relación al ítem de acceso a puestos de trabajo se obtuvo como resultado según las opiniones de los entrevistados, que el $60 \%$ de ellos cree que el género si tiene una influencia al momento de elegir a una persona en un puesto de trabajo, que no represente un cargo directivo, en el área de Terapia Ocupacional. Según lo referido po os profesionales esta influencia generalmente está dada por el contexto en el que se desarrolla el puesto de trabajo y la población a la que va dirigida la intervención.

"Aunque a veces uno necesita el perfil, como perfil necesita un varón...yo he visto si algunos avisos así como "se requiere Terapeuta ojalá varón" (Ent: 1)

"En los cargos en donde yo he visto en TO esta ¿diferenciación de género? Son cargos de donde uno trabaja con población de alto riesgo, donde vas a trabajar en la calle, en una población, en un no sé módulo de alta complejidad en la cárcel y ahí entra el hombre no la mujer (Ent: 4)

A su vez el $20 \%$ de los entrevistados plantea que para la contratación de un Terapeuta Ocupacional se visualizan las particularidades que requiere el puesto en relación a las características asociadas culturalmente a un determinado género.

"Haber cargos dónde trabajas con adultos que tienen muchas dificultades de movimiento y pareciera que ser un hombre tiene mayor posibilidad por la fuerza, con esta idea del hombre fuerte, entonces aparece el estereotipo en la postulación al cargo" (Ent: 4)

"Mira, específicamente cuando entré a trabajar al hospital, el clima laboral, en este contexto, me habían comentado que era complejo, por lo tanto me llamaron específicamente porque por ser TO varón tenía también otra forma de hacer, de poder como desenvolverme" (Ent: 10)

En relación a la influencia en el desempeño en relación al acceso a los cargos directivos por género, e $20 \%$ de las mujeres entrevistadas, menciona que si hay un impacto principalmente por los roles asociados a su género como lo es el rol de madre o la responsabilidad de 
las tareas domésticas, que en la actualidad son asumidos más frecuentemente por las mujeres que por los varones.

Por otro lado ninguno de los Terapeutas varones entrevistados ha visto influencia en su desempeño en este aspecto.

\section{Estatus de minoría}

En este ítem se analizaron el efecto de autoridad asumida que se va a entender como el efecto que puede ocurrir cuando en un grupo de trabajo con predominancia del género femenino, los sujetos del género masculino toman un rol de liderazgo, estando ambos géneros en las mismas condiciones y en una simetría jerárquica en cuanto a su puesto de trabajo y el efecto de consideración especial, que apunta que los varones son favorecidos de su estatus de minoría producto de un trato diferencial recibido (Simpson, 2004).

Con respecto al primer efecto, el $40 \%$ de los participantes considera que el liderazgo por parte del hombre está mediado por la cultura y una sociedad machista, además indican que éstos cumplen un rol de mediador en equipos donde predominan las mujeres.

"Efectivamente como somos una sociedad fundamentalmente muy machista, el hombre si siempre se ha creído con mayor capacidad para liderar equipo, este como estereotipo del hombre que tiene capacidad de dirigir, cierto, de dirigir personas, de tomar decisiones (...) bruscas y serias y agresivas, cuando son necesarias (...) son características súper asociadas a lo masculino" (Ent: 2)

"Incluso los equipos de mujeres buscan también hombres que se incorporen dentro del equipo, porque ellas mismas también reconocen, por lo menos me han reconocido a mí, que necesitan esta de repente visión un poquito más sistémica y rígida en el fondo" (Ent:7)

Sin embargo el $60 \%$ de los participantes relata que este efecto de autoridad asumida no se da por un tema de género, sino que responde a otros aspectos, como lo son las características personales y las circunstancias en las que se da la situación.
"Creo que va a depender de las características de la persona, no depende si es hombre o mujer, porque me ha pasado ver hombres que son más pasivos y he visto mujeres que tienen este rol más activo más, más de líder" (Ent: 8)

Con respecto al efecto de consideración especial, el $20 \%$ de los entrevistados mencionan que si perciben un trato diferenciado dado por el género, específicamente hacia la minoría masculina, ya sea por parte de la población a intervenir o por los propios colegas con los cuales se relacionan en el área laboral.

"Lo que yo he visto normalmente es que si se hacen características o consideraciones especiales a los hombres por pertenecer a una minoría que tiene como una mirada positiva" (Ent: 2)

"Ahí hay ciertos grupos, por ejemplo, adultos mayores mujeres, que tienen mayor afín (...) en el caso de los hombres como que de alguna forma somos más regalones por este grupo de usuarios" (Ent: 10)

Por el contrario, el $80 \%$ de la muestra de estudio no concuerda con la teoría indicada anteriormente, lo señalado anteriormente se ve reflejado en lo exhibido por los siguientes relatos:

"Yo creo que más que determinar si es un hombre o una mujer lo determinante tiene que ver con las características personales, que puede influir, o positivamente o negativamente" (Ent: 1)

"Es que si tú me dices que es asociado al género, yo te podría decir que no, si tú me dices que es asociado como a las características como personales, yo te podría decir que sí, pero no sé si mis características personales responden a mi género" (Ent: 7)

Con respecto a la influencia del género en su desempeño laboral, en relación al efecto de autoridad asumida, el 20\% de los Terapeutas Ocupacionales varones refieren que en el ámbito laboral hay circunstancias que le afectan en menor medida que sus colegas mujeres señalando tener una mayor capacidad de tolerancia al 
estrés favoreciendo de esta forma su desempeño en el trabajo. Por otro lado, el 20\% de las mujeres entrevistada señalaron que las diferencias en el trato entre los colegas fomentarán situaciones negativas en su clima laboral afectando así su desempeño.

\section{DisCUSIÓN}

El estudio "Terapeutas Ocupacionales: influencia de género en su desempeño laboral", nace bajo el interé de analizar la influencia del género en el desempeño aboral de estos profesionales, bajo la hipótesis de que el género si influye de alguna u otra forma en el desempeño profesional de un grupo de Terapeutas Ocupacionale de la ciudad de Temuco.

Con respecto a la hipótesis planteada acerca de si el desempeño laboral se ve afectado por el género se concluye que ésta sí se cumple, ya que a través del análisis de las determinantes estudiadas asociadas género se identificó que algunas de ellas se presentan en la realidad en mayor medida que otras, influyendo de esta manera en el quehacer profesional. Además se descubrió la existencia de variables que influyen en el desempeño laboral pero que no están asociadas al concepto de género.

En relación a los objetivos específicos planteados, fue posible conocer las determinantes laborales que se ven influenciadas por el género en el desempeño de los Terapeutas Ocupacionales así como también se compararon tales determinantes entre hombres y mujeres Terapeutas Ocupacionales, dando cumplimiento al objetivo genera correspondiente a determinar la influencia del género en el desempeño laboral de los Terapeutas Ocupacionales.

Es así como podemos evidenciar que en el conglomerado de Terapeutas Ocupacionales entrevistados la gran mayoría mencionó que el concepto de género influye en alguna medida en el ejercicio de la profesión dando cumplimiento al objetivo general planteado en la investigación. Cabe destacar que este fenómeno no está establecido por la disciplina, sino que por la sociedad en la que se encuentran inmersos, por lo que se puede concluir que el contexto toma gran importancia al momento de desempeñar una actividad laboral, el cual conserva rasgos de una visión estereotipada acerca de los roles que deben desempeñar las personas, traspasando así los muros de la profesionalidad.

A pesar de que el estudio responde a la pregunta de investigación, el equipo considera que aún existen preguntas sin responder con respecto al fenómeno social de género en nuestra realidad, entre las cuales se encuentran: ¿Existirá otra categoría aparte de género que influya en el desempeño laboral de los Terapeutas Ocupacionales? ¿Existirán otras variantes de género que influyen en el desempeño laboral? ¿Existe igualdad de los derechos para hombres y mujeres en el área laboral? ¿Es necesario mantener las diferencias entre hombres y mujeres? ¿En qué área laboral se encuentra más presente la influencia del género?

El grupo de investigadores observa que los Terapeutas Ocupacionales entrevistados en su gran mayoría ven las brechas de género alejadas de su realidad laboral, no teniendo conciencia de que tales situaciones pueden ocurrir en sus circunstancias de trabajo en primera instancia.

Finalmente se puede decir que el tema de estudio es de gran relevancia para la profesión ya que se analizó la realidad y el contexto en el que los Terapeutas Ocupacionales se desempeñan, teniendo una mirada crítica de los fenómenos derivados del género, el que constituye una categoría de análisis transversal y que es inherente al ser humano. Es por lo anterior que es importante exponer los datos que se tienen de la realidad de estos profesionales ya que por medio de este estudio que constituye un tema de génesis investigativa se podrán visualizar las necesidades diferenciadas entre hombres y mujeres Terapeutas Ocupacionales.

Así mismo, es importante destacar que los resultados obtenidos en esta investigación constituyen la piedra angular de futuros estudios que relacionen la disciplina de Terapia ocupacional y Perspectiva de Género. 


\section{ReferenCias BibliográficAs}

Asociación Americana de Terapia Ocupacional (AOTA). (2010) Marco de Trabajo para la Práctica de Terapia Ocupacional: Dominio y Proceso. Recuperado de: http://www.terapia-ocupacional.com/ aota2010esp.pdf.

Asociación de Hombres por la lgualdad de género (AHIGE), (2011). Quiénes somos, Principios básicos. Recuperado de http://www.ahige.org/ principiosbasicos.html.

Chávez, J., C. (2004) Perspectiva de Género. México. UNAM Plaza y Valdés. Colegio de Terapeutas Ocupacionales de Chile A.G. (2014). Antecedentes sobre la formación universitaria de Terapeutas Ocupacionales en Chile. Recuperado de http://www.terapia-ocupacional.cl/documentos/ miscelaneo/2014_ANTECEDENTES_COLTO_2014.pdf.

Departamento de Estadísticas e Información de Salud. (2012). Profesionales y Técnicos del Sector Salud, por sexo, registrados en la Superintendencia de Salud. Chile. Recuperado dehttp://www.deis. cl/wp-ontent/uploads/2013/04/Profesionales-y-técnicos-del-sector-salud-dic-2012.xls.

Facio, A., y Fries, L. (2005). Feminismo, género y patriarcado. ACADEMIA. REVISTA SOBRE ENSEÑANZA DEL DERECHO DE BUENOS AIRES, (6), 262

Macera, R. (2015) Género y mercado laboral: ¿Por qué las mujeres ganan menos que los hombres? Pontificia Universidad Católica De Chile. Recuperado de: http://escueladeadministracion.uc.cl/directorio/ columnas/decima-columna-de-la-seccion-general-management-escrita-por-la-profesora-rosario-macera-y-publicada-el-sabado-21-de-noviembre-en-el-mercurio/

Sendotu. (2010). Intervención social desde el enfoque de género. Recuperado de http://www.trabajosocialleon.org/documentos/circulares_40_1383044941.pdf.

Simpson, R., (2004). Masculinity at work: The experiences of Men and Female Dominated Occupations. Work Employment and society, 18(2), 1-35.

Todaro, R., Godoy, L., Abramo, L. (2001). Desempeño laboral de hombres y mujeres: opinan los empresarios. Recuperado de: http://www. scielo.br/pdf/cpa/n17-18/n17a08.

Unión Europea. (2014). Cómo Combatir la Brecha Salarial entre Hombres y Mujeres en la Unión Europea. Recuperado de http://ec.europa.eu/ justice/gender-equality/files/gender_pay_gap/140319_gpg_es.pdf.

Vasilachis, I. (2009). Estrategias de investigación cualitativa. Barcelona España: Editorial Gedisa, S.A 